\title{
The evaluation of the retrobulbar orbital fat tissue and optic nerve with strain ratio elastography
}

\author{
Murat Vural $^{1}$, Dilek Acar¹, Uğur Toprak¹, Mehmet Numan Alp², Özlem Gürbüz Köz², \\ Bige Sayın', Gizem Kuşçuoğlu Abat ${ }^{1}$
}

${ }^{1}$ Department of Radiology, ${ }^{2}$ Department of Ophthalmology, Ankara Numune Training and Research Hospital, Ankara, Turkey

\begin{abstract}
Aim: The aim of this study was to evaluate the diagnostic performance of strain ratio elastography in the assessment of retrobulbar orbital fat tissue. Materials and methods: The retrobulbar fat tissue of 14 eyes in 14 participants was scanned by sonoelastography. All the participants had permanent vision loss secondary to glaucoma in at least one eye. The elasticity scores were determined and the strain ratio was based on the comparison of the average strain measured in the retrobulbar fat tissue around the optic nerve at the same depth. Results: The mean strain values for the optic nerve (B) and the retrobulbar fat tissue (A) were $0.78 \pm 0.61$ and $1.43 \pm 0.99$, respectively ( $\mathrm{p}=0.005$ ). The mean strain ratio (B/A) was $0.56 \pm 0.24$. Conclusion: This preliminary study showed that by providing strain values and ratios of different orbital tissues sonoelastography may have a potential use in the diagnosis and follow-up of orbital inflammatory and infiltrative diseases.
\end{abstract}

Keywords: sonoelastography, retrobulbar fat, optic nerve

\section{Introduction}

Gray-scale ultrasound is a common and noninvasive tool that has been used for many years to observe bulbus oculi and retrobulbar fat. Although, elastography is a new sonographic technique, the evaluation of orbital diseases using this method has not been found to be as widespread as its use in other organ systems. Recently, elastography has been frequently used in the diagnosis of breast, thyroid, lymph node, muscular, and abdominal solid organ diseases [1-5].

Principally, sonoelastography depends on the measurement of the level of tissue deformation as a result of compression. There are two basic ultrasonic elastography methods namely; free-hand method and shear-wave method. They have drawn more attention in recent years

Received 22.09.2014 Accepted 05.11.2014

Med Ultrason

2015, Vol. 17, No 1, 45-48

Corresponding author: Murat Vural

5/15 Huseyin Rahmi street, Cankaya

Ankara,Turkey, 06680

Phone: +90 5323173355

Fax: +90 3123126876

E-mail: mvura199@hotmail.com after studies demonstrated that there is a close relation between the elasticity and histological characteristics of tissues [6]. In elastography, a color map is superimposed on the real-time grayscale ultrasound image, in which each color represents a certain level of tissue deformation.

Free-hand method, also known as strain ratio elastography, is a semi-quantitative method that reveals the hardness of tissue. This method compares the difference between the compliances of target area A and target area B. In strain elastography, one tissue is chosen as the reference point. The difference between the strain values of the other tissues that are assumed to be diseased is calculated according to the reference tissue.

In the literature, there is only one orbital sonoelastography study in which the color-coded transverse sonoelastographic images of five patients were obtained using Free-hand elastography. The luminosity signal of the structures was measured (histogram, luminosity channel) using a commercial program. According to their color codes, the strain ratios of the retrobulbar fat tissue and optic nerve were calculated indirectly, the direct values were not given by the ultrasound equipment [7]. 
Retrobulbar fat tissue changes can develop from many causes such as infection, inflammation, and neoplastic diseases. Computerized tomography (CT) has a limited application in the follow-up of these patients, since it has ionizing radiation. Magnetic resonance imaging (MRI) offers high resolution images; however, it is time consuming, requires an experienced radiologist and costs more than other techniques. There is a need for a more practical method to evaluate the retrobulbar area in both the initial diagnosis and follow-up of the patients. In this paper retrobulbar elastography is presented as a new imaging method for ocular examinations using the optic nerve as a reference area.

\section{Materials and methods}

\section{Patients}

U.S. Food and Drug Administration has not approved free-hand orbital sonoelastography for routine ophthalmic examinations. The participants in this study had unilateral permanent vision loss due to end stage glaucoma. Besides, the examination was only performed on each participant's eye that had a complete loss of vision.

Fourteen participants who had permanent vision loss at least in one eye secondary to glaucoma were recruited for this study from February 2013 to December 2013. Two of the participants who had bulbus oculi deformation and optic nerve thickening, respectively, were excluded from the study. Thus the statistical analysis was performed on 12 participants. Each participant underwent retrobulbar elastography for the assessment of retrobulbar fat and optic nerve strain values. The study was approved by the Ethics Committee of the institution, and all participants gave informed consent to participate in the study.

\section{Ultrasonography}

The B-mode ultrasonography and sonoelastography of all volunteers were performed by the same operator. All the images were acquired using HI VISION Avius color Doppler ultrasonography with elastography software and a 4-9 $\mathrm{MHz}$ linear transducer (Hitachi Medical, Tokyo, Japan). A coupling gel was used between the probe and the eyelids to obtain images. Participants were instructed to keep their eyelids closed and not to move their eyes during the examination. The elastographic images were generated using freehand manual compression. The region of interest (ROI) extended from the bulbus oculi to the posterior portion of retrobulbar fat tissue. A light compression was applied after detecting the optic nerve. The pressure indicator bar showed a value of 2 or 3 , and the mean speed of the probe movement was once or twice compressions per second. The elasticity image was displayed with color mapping for each pixel accord-

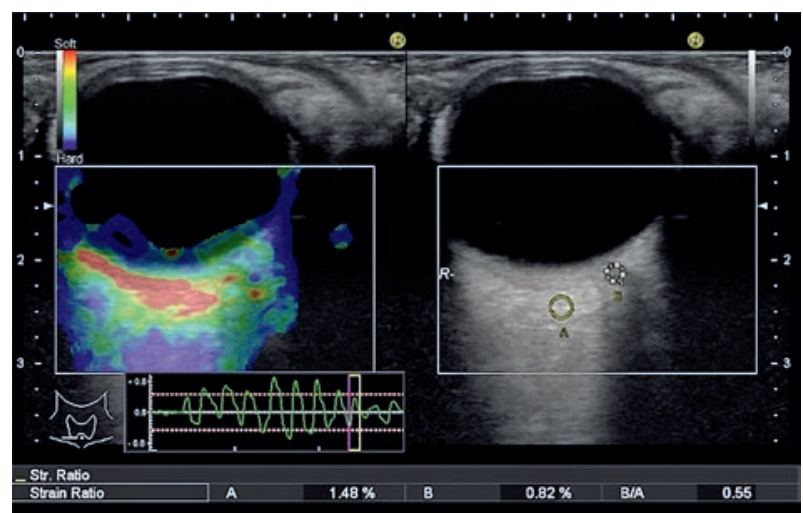

Fig 1. Orbital strain elastography image shows the color map and real-time grayscale ultrasound. The pressure indicator bar shows both the number and strength of the repetative compressions applied by the ultrasound probe.

ing to the degree of strain within the ROI. On the color scale, a red color represented the softest component, while a green and blue color represented the intermediate and hardest components, respectively (fig 1).

The calculation of the strain ratio (SR) was based on the comparison of the strains measured in the optic nerve and the adjacent retrobulbar fat tissue at the same depth. The operator manually drew the contours. First the optic nerve was selected and labeled as A, then the adjacent retrobulbar fat tissue region of the same depth was selected and labeled as B. The stiffness of the tissues were represented by strain ratio (B/A) which was calculated by sonographic equipment.

Statistical analysis

The data analysis was performed in commercial code SPSS for Windows, version 11.5 (SPSS Inc., Chicago, IL, United States). Whether the distributions of continuous variables were normal was determined by a Shapiro Wilk test. Data was shown as mean \pm standard deviation (SD) or number of cases and percentage (\%), where applicable. Whether, the mean difference between the strain values of optic nerve and retrobulbar fat tissue was statistically significant was evaluated by a Paired t-test. The degrees of association between continuous variables were calculated by Spearman's correlation coefficient. A Student's $t$ test was applied to determine the differences between both age and gender regarding the strain ratio. A $p$ value less than 0.05 was considered statistically significant.

\section{Results}

The mean age of the 12 participants $(5$ male, $7 \mathrm{fe}$ male) was $64.7 \pm 15.2$ (ranging from 35 to 87 ) years. The mean strain value of optic nerve (B) was $0.78 \pm 0.61$, and the retrobulbar fat tissue (A) was $1.43 \pm 0.99$. The retrob- 
ulbar fat tissue strain value was significantly higher than the respective value for the optic nerve $(\mathrm{p}=0.005)$ (fig 2 ). The mean strain ratio (B/A) was $0.56 \pm 0.24$. In addition, the strain values of the retrobulbar fat tissue and optic nerve had a statistically significant correlation $(\mathrm{R}=0.706$ and $\mathrm{p}=0.010$ ) (fig 3). Strain ratio (B/A) was $0.52 \pm 0.29$ in females and $0.60 \pm 0.21$ in males. The strain ratio showed no statistically significant difference according to gender $(\mathrm{p}=0.577)$. In addition, there was no significant correlation between the age and strain ratio $(\mathrm{R}=0.399$ and $\mathrm{p}=0.199)$.

\section{Discussions}

In this study, normal strain values of retrobulbar fat tissue and optic nerve were detected in the participant population that did not have any neoplastic or inflammatory retrobulbar diseases. The main result of this study is that strain value of retrobulbar fat tissue is definitely different from the value obtained from the optic nerve. In relation to the infiltration of retrobulbar fat tissue with inflammatory and neoplastic processes, strain value will decrease and become closer to that of the optic nerve. This result has the potential to be used in the initial diagnosis and follow-up of the patients with retrobulbar fat tissue diseases. Since neither gender nor age had a statistically significant affect on the correlation between strain values of the retrobulbar fat tissue and the optic nerve, the evaluation of the orbital structures would become easier by this method.

There are many inflammatory diseases of the orbit including Graves' disease, orbital cellulitis, and pseudotumor, in which the earliest changes may be a subtle edema of the retrobulbar fat. Orbital muscle and fat, optic nerve and its sheath could be a major immunological target for some diseases such as thyroid orbitopathy $[8,9]$.

Many tumors of the orbit; optic gliomas, optic nerve sheath meningiomas, cavernous hemangiomas, and lymphangiomas have specific imaging features. Thus it is often possible to arrive at a limited differential diagnosis and clearly suggests the most likely histology by correlating these features with the age of the patient and the location of tumor within the orbit.

Sonoelastography is a diagnostic tool which can be used to evaluate tissue elasticity and hardness in differentiating pathological (benign, malignant) lesions from normal tissues [10]. The elasticity of pathological tissue decreases according to adjacent normal tissue. In breast lesions a pathological lesion and normal breast tissue are compared and according to different elastic scores, histopathological diagnosis can be predicted since the hardness of malignant lesions is greater than that of benign ones. In the present study, with the semi-quantitative

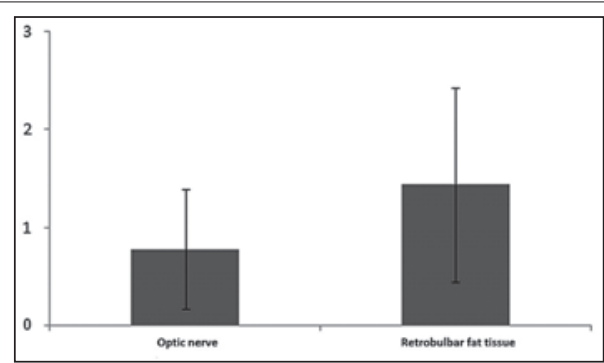

Fig 2. Strain values of the optic nerve and retrobulbar fat tissue.

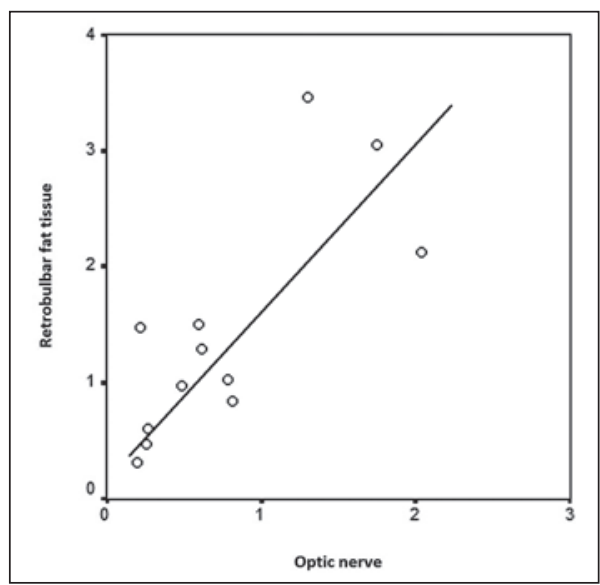

Fig 3. Strain relations of the optic nerve and retrobulbar fat tissue.

evaluation of the sonoelastographic images, the strain ratio of the retrobulbar tissue was calculated using the strain value measurement of the adjacent optic nerve as an internal reference. In this way, it is assumed that any neoplastic and inflammatory disease decreases the elasticity and strain value of this tissue; thus it can be diagnosed by elastography.

Repetitive compressions are applied to the bulbus oculi with an ultrasound probe to detect the elasticity of the optic nerve and retrobulbar fat tissue. In this study, the length of the ultrasound probe was $50 \mathrm{~mm}$ and the width was $14 \mathrm{~mm}$. At each impulse of the ultrasound probe for the strain elastography procedure, the total pressure exerted by the probe to the eye is approximately equal to a weight of 50-60 gr. It is accepted that another risk factor for strain elastography is the heating of the tissues by the secondary acoustic radiation [11]. However, the total amount of the time for the examination of one eye was 2 to 3 seconds. Therefore, it would not cause any complications regarding excessive heat since the duration of examination was short.

All participants in this study had permanent vision loss in at least one eye caused by optic atrophy secondary to glaucoma. This might have changed the elasticity 
of the optic nerve to some extent. There were different strain ratios between optic nerve and retrobulbar fat tissue according to the results. However, the strain ratios of healthy optic nerves and atrophic nerves were not compared and this can be considered a limitation of this study. In the published literature there was only one study with five patients [7]. It is believed that retroortbital fat tissue and optic nerve sonoelastography should be evaluated in larger-scale studies at normal populations.

In conclusion, the participants in this study had glaucomatous blind eyes and orbital elastography was able to differentiate the strain values of the optic nerve and retrobulbar fat tissue. When the retrobulbar fat tissue elasticity is equal to or lower than that of the optic nerve, it may be assumed that there is a pathology in the retrobulbar fat tissue. After the appearance of new studies with normal populations, the strain ratio of retrobulbar fat detected by orbital elastography, has the potential to be used as a new technique for early detection and follow-up of the inflammatory and neoplastic diseases of the retrobulbar fat tissue.

Conflict of interest: none

\section{References}

1. Ghajarzadeh M, Sodagari F, Shakiba M. Diagnostic accuracy of sonoelastography in detecting malignant thyroid nodules: a systematic review and meta-analysis. AJR Am J Roentgenology 2014; 202: 379-389.
2. Sandulescu L, Rogoveanu I, Gheonea IA, Cazacu S, Saftoiu A. Real-time elastography applications in liver pathology between expectations and results. J Gastrointestin Liver Dis 2013; 22: 221-227.

3. Botar Jid C, Vasilescu D, Damian L, Dumitriu D, Ciurea A, Dudea SM. Musculoskeletal sonoelastography. Pictorial essay. Med Ultrason 2012; 14: 239-245.

4. Grajo JR, Barr RG. Strain elastography for prediction of breast cancer tumor grades. J Ultrasound Med 2014; 33: 129-234.

5. Tatar IG, Kurt A, Hekimoglu B. Advantages and pitfalls of elastosonography in the evaluation of cervical lymph nodes. Med Ultrason 2013; 15: 333-334.

6. Thitaikumar A, Ophir J. Effect of lesion boundary conditions on axial strain elastograms: a parametric study. Ultrasound Med Biol 2007; 33: 1463-1467.

7. Detorakis ET, Drakonaki EE, Tsilimbaris MK, Pallikaris IG, Giarmenitis S. Real-time ultrasound elastographic imaging of ocular and periocular tissues: a feasibility study. Ophthalmic Surg Lasers Imaging 2010; 41: 135-141.

8. Bahn RS. Graves' ophthalmopathy. N Engl J Med 2010; 362: 726-738.

9. Karesh JW, On AV, Hirschbein MJ. Noninfectious orbital inflammatory disease. In: Tasman W, Jaeger EA (eds). Duane's Ophthalmology. 15th ed. Philadelphia, Pa. Lippincott Williams \& Wilkins 2009: 245-296.

10. Barr RG, Destounis S, Lackey LB, Svensson WE, Balleyguier C, Smith C. Evaluation of breast lesions using sonographic elasticity imaging: a multicenter trial. J Ultrasound Med 2012; 31: 281-287.

11. Duck FA. Medical and non-medical protection standards for ultrasound and infrasound. Prog Biophys Mol Biol 2007; 93: 176-191. 